\title{
Carving bipolarity using a lithium sword
}

\section{Gin S. Malhi and John R. Geddes}

\section{Summary}

The classification of mood disorders lacks precision and consequently there has been no recent meaningful advance in their treatment. By virtue of its therapeutic specificity, lithium responsivity offers an opportunity to diagnose a definitive subtype of mood disorders that may provide a platform for the development of targeted therapy.

\section{Declaration of interest}

G.S.M. is a chief investigator on an NHMRC Program Grant. He has also received research support from AstraZeneca, Eli Lilly, Organon, Pfizer, Servier and Wyeth; has been a speaker for AstraZeneca, Eli Lilly, Janssen-Cilag, Lundbeck, Pfizer, Ranbaxy, Servier and Wyeth; and has been a consultant for AstraZeneca, Eli Lilly, Janssen-Cilag, Lundbeck and Servier. J.G. is a National Institute for Health Research (NIHR) senior investigator.
Professor Gin S. Malhi (pictured) is Head of the Department of Psychiatry at the University of Sydney, and Clinical and Research Director of the CADE Clinic at Royal North Shore Hospital where he cares for patients with mood disorders, in particular bipolar disorder. He is also Editor in Chief of the Australian and New Zealand Journal of Psychiatry and Executive Directo of the University of Sydney Advanced Research Clinical High-field Imaging (ARCHI) facility. Professor John R. Geddes is Head of the Department of Psychiatry at Oxford University, and Honorary Consultant Psychiatrist at the Oxford Health NHS Foundation Trust where he provides clinical care for patients with mood disorders, specialising in bipolar disorder. He is also Director of R\&D at the Oxford Health NHS Foundation Trust.

\section{Diagnostic problems caused by polarity}

The conventional clinical picture of bipolar disorder is that of an illness characterised by discrete episodes of mania and depression, sometimes alternating, but often not. The occurrence of mania separates bipolar disorder from major depression, and in essence defines the illness. However, undue emphasis on polarity has created a number of serious diagnostic problems.

First, the fact that 'mixed states' commonly occur in clinical practice, and by definition do not conform to either syndrome of depression or mania, challenges the fundamental validity of an axial model of bipolar disorder with diametrically opposing mood states. ${ }^{1}$ Second, diagnostic weighting of polarity in classification systems has unwittingly diverted clinical attention away from other more important aspects of affective disorders such as their longitudinal pattern, in particular, the prominence of interepisodic symptoms and mood instability and the recurrence of mood episodes over the course of the illness. Third, the lack of clinical validity of a classification of mood disorders based on polarity has given impetus to the development of spectrum models, which lack purpose with respect to treatment.

In an attempt to iron out the diagnostic wrinkles caused by DSM-IV ${ }^{2}$ mixed episodes, DSM $-5^{3}$ removed their codification altogether and introduced a mixed features specifier, which can be used to nuance mood episodes when deemed necessary. But reconfiguring the classification of mixed states in this manner does not explain their aetiology, and the overarching paradigm for mood disorders based on antipodal phases of affective illness remains incomplete and unrealistic; after all, it is impossible to conceive of a mental state in which an individual's mood is simultaneously elevated and depressed. ${ }^{4}$

In contrast to mixed episodes, which should not have been included in DSM-IV, a lack of emphasis on the longitudinal pattern of affective disorders is an error of omission. This is because the course of mood disorders is an important feature that meaningfully groups subtypes and informs treatment. For example, the classic diagnosis of manic-depressive illness contained all patients with recurrent mood episodes irrespective of polarity. But this emphasis on recurrence was lost when bipolar disorder was formalised in DSM-III ${ }^{5}$ and polarity gained primacy. Hence modern-day bipolar disorder is not synonymous with manic-depressive illness and this is evident with respect to treatment. ${ }^{6}$

The categorical classification of mood disorders has proven to be problematic. For example, clinically an episode of depression within major depressive disorder is indistinguishable from that within bipolar disorder, but their treatment responses differ. Within bipolar disorder, definition of the type II bipolar subtype rests on identifying a moderate form of mania, but the upper and lower duration cut-offs used to delineate hypomania are completely arbitrary, and have been shown to have no clinical or biological correlates. Indeed, in practice, periods of manic symptoms of fewer than 4 consecutive days' duration are common and can cause as much disability as longer episodes of illness. Similarly, the transition from hypomania to mania on the basis of loosely defined 'marked impairment' and/or a consequence of illness, namely 'hospitalisation', lacks credence. In part because of these difficulties, but also because symptoms appear to manifest dimensionally, the concept of a spectrum, both in terms of symptoms and disorders, is intrinsically appealing; but it too has failed to provide a better understanding of mood disorders and advance clinical management.

Fundamentally, the inadequacies of contemporary diagnostic systems ${ }^{7}$ highlight our sciolism concerning the pathophysiology of bipolar disorder. Yet we can probably do much better if we move away from clinical assessment based on retrospective anamnesis and towards detailed, prospective measurements using the big data delivered by pervasive modern communication technologies and wearable devices. ${ }^{8}$ Such deep phenotyping is likely to yield insights into underlying neurobiology and to aid the development of treatments for bipolar disorder. It is worth noting that, despite heightened interest in bipolar disorder, its pharmacological treatment remains woefully suboptimal, with no new medications being developed specifically for the treatment of mania or bipolar depression since the advent of lithium. ${ }^{9}$

\section{Lithium's cutting edge}

To achieve a more meaningful taxonomy of mood disorders, and to develop better treatments, a new diagnostic paradigm and a 
different approach to treatment development is necessary. Traditionally, psychiatric disorders have been diagnosed according to signs and symptoms on the assumption that these form relatively homogeneous groups, which lend themselves to systematic investigation. But, in actuality, psychiatric diagnoses have generated loosely defined categories with composite aetiologies, and this has made it difficult to determine their pathophysiology. An alternative approach is to use treatment response to guide us to underlying processes - a reverse translation.

In mood disorders, lithium has both antimanic and antidepressant properties, but its effects when treating acute episodes are slow, for example, in comparison with secondgeneration antipsychotics. ${ }^{10}$ In contrast, its effectiveness longer term, and in particular with respect to prophylaxis, is unparalleled. ${ }^{11}$ Clinically, this is its most important effect and one that confers a significant advantage. Lithium's specific action of mood stabilisation is its cutting edge.

\section{Defining lithium response}

Longitudinal studies that have examined the clinical effects of lithium have managed to characterise those patients with mood disorder most likely to respond. The clinical profile of an individual who responds to lithium is essentially that of someone with 'classic' manic-depressive illness; namely, recurrent unipolar and bipolar mood disorders in which periods of illness are clearly episodic with complete remission in between. Furthermore, individuals who are lithium responders have low psychiatric comorbidity and are more likely to have first-degree relatives with lithium-responsive manic-depressive illness, suggesting that responder status runs in families. ${ }^{12}$ This inference has gained further support in the findings of a recent longitudinal bipolar trajectory study, which noted that the offspring of lithium responders were more likely to have an episodic clinical course of illness with complete remission in between compared with the offspring of lithium non-responders. ${ }^{13}$

The action of lithium in the treatment of classic mania is often evident within the first week of treatment, and this early response is predictive of ongoing improvement culminating in remission. ${ }^{14}$ And in practice, this is useful because it allows the prompt recognition of those likely to benefit from lithium treatment. Clinically, approximately a third of patients with bipolar disorder treated with lithium have a robust response and qualify as lithium responders. Lithium response can be measured using a two-part scale, ${ }^{11}$ which assesses illness response and the degree to which this is attributable to lithium treatment. The scale provides both categorical and dimensional ratings and has been used mainly retrospectively. This is because prospective assessment requires a significant follow-up period, usually several years, so as to allow meaningful comparison of pre-lithium and lithium treatment periods, or between lithium and a comparator in clinical trials on measures of relapse and recurrence. Therefore, defining individuals as lithium responders is necessarily difficult and made more so by the intrinsic unpredictability of the illness and added complexity of extrapolating findings from research on groups of patients to individuals in clinical practice.

We therefore need sensitive and specific biomarkers that allow early and accurate prediction of therapeutic response to lithium; this is pivotal to precision medicine and holds potential to advance treatment outcomes in psychiatry. ${ }^{15}$ Genes may be one such biomarker as shown in a recent study, which reported associations between single nucleotide polymorphisms (SNPs) in genes involved in glutamatergic neurotransmission and lithium response during maintenance treatment of patients with type I bipolar disorder. ${ }^{16}$ However, these findings require corroboration and await replication. ${ }^{17,18}$ Similarly, investigation of the shortterm effects of lithium on neural systems using modern imaging and cognitive neuroscience - and relating this to the effects on mood instability - may provide the traction required to generate usable biomarkers and intermediate outcomes.

Alongside its profound mood stabilising effect, lithium has striking anti-suicidal ${ }^{19}$ and neuroprotective properties, which further distinguish those patients that respond. For example, telomere shortening reflects oxidative stress and inflammation and is a reliable marker of ageing. It is also implicated in mood disorders and can be countered by the actions of antidepressants. In a recent study examining the effects of lithium on telomere length in bipolar disorder, lithium was found to have a protective effect, which correlated closely with its clinical efficacy. ${ }^{20}$ However, despite its clinical benefits, lithium is potentially toxic at double the therapeutic dose and has poor long-term tolerability for many patients. ${ }^{21}$ There is certainly room for improvement, and there needs to be an energetic search for lithium mimetics that may be able to replicate the efficacy of lithium without reproducing its side-effect problems. The search for analogues is impeded by our uncertainty about lithium's precise molecular mechanism of action, $^{22}$ but among the many potential targets, inositol monophosphatase is probably the best current candidate site of action. ${ }^{22}$ A recent search of drug libraries for agents with a profile akin to lithium, identified ebselen, an antioxidant and inositol monophosphatase inhibitor with established safety ${ }^{23}$ and, excitingly, this molecule has been successful in current best animal models of bipolar disorder. The next step is to assess its effects in patients.

\section{Conclusions}

Lithium remains the best pharmacological agent for the long-term treatment of recurrent mood disorders, in particular, bipolar disorder. It has specific actions, which need to be better understood to allow development of more targeted treatments for mood disorders. Elucidating the precise effects of lithium at clinical and molecular levels is likely to aid further clinical and basic research into bipolar disorder and to drive forward much needed drug discovery. Specifically, defining a lithium-responsive subtype within mood disorders will provide a cornerstone for the construction of a therapeutically meaningful taxonomy. The clinical circumscription of lithium responders will introduce greater homogeneity both within the taxon and in non-lithium responsive mood disorders outside of this group. It is time to return to lithium, both for its clinical benefits and for the scientific potential provided by close study of its effects. We should take advantage of the unique properties of lithium - to help redefine mood disorders and to discover new targets for treatments based on a deep understanding of biology.

Gin S. Malhi, Department of Psychiatry, University of Sydney, Royal North Shore Hospital, Sydney, Australia; John R. Geddes, Department of Psychiatry, University of Oxford, Warneford Hospital, Oxford, UK

Correspondence: Gin S. Malhi, CADE Clinic, Level 3 Main Building, Royal North Shore Hospital, St Leonard's NSW 2065, Sydney, Australia. Email: gin.malhi@sydney.edu.au

First received 2 Jun 2014, final revision 21 Aug 2014, accepted 9 Sep 2014

\section{References}

1 Malhi GS. Diagnosis of bipolar disorder: who is in a mixed state? Lancet 2013; 381: 1599-600. 
2 American Psychiatric Association. Diagnostic and Statistical Manual of Mental Disorder (4th edn) (DSM-IV). APA, 1994

3 American Psychiatric Association. Diagnostic and Statistical Manual of Mental Disorder (5th edn) (DSM-5). APA, 2013.

4 Malhi GS, Lampe L, Coulston CM, Tanious M, Bargh DM, Curran G, et al. Mixed state discrimination: a DSM problem that won't go away? J Affect Disord 2014; 158: 8-10.

5 American Psychiatric Association. Diagnostic and Statistical Manual of Mental Disorder (3rd edn) (DSM-IIII). APA, 1980.

6 Goodwin FK, Jamison R. Manic-Depressive IIIness: Bipolar Disorders and Recurrent Depression. Oxford University Press, 2007.

7 Malhi GS. DSM-5: ordering disorder? AuS NZ J Psychiatry 2013; 47: 7-9.

8 Bopp JM, Miklowitz DJ, Goodwin GM, Stevens W, Rendell JM, Geddes JR. The longitudinal course of bipolar disorder as revealed through weekly text messaging: a feasibility study. Bipolar Disord 2010; 12: 327-334.

9 Geddes JR, Miklowitz DJ. Treatment of bipolar disorder. Lancet 2013; 381: $1672-82$.

10 Cipriani A, Barbui C, Salanti G, Rendell J, Brown R, Stockton S, et al. Comparative efficacy and acceptability of antimanic drugs in acute mania: a multiple-treatments meta-analysis. Lancet 2011; 378: 1306-15.

11 Geddes JR, Goodwin GM, Rendell J, Azorin JM, Ostacher MJ, Morriss R, et al Lithium plus valproate combination therapy versus monotherapy for relapse prevention in bipolar I disorder (BALANCE): a randomised opn-label trail. Lancet 2010; 375: 385-95.

12 Grof $P$, Duffy A, Carazzoni P, Grof E, Garnham J, MacDougall M, et al. Is response to prophylactic lithium a familial trait? J Clin Psych 2002; 63 942-7.
13 Duffy A, Horrocks J, Doucette S, Keown-Stoneman C, Mccloskey S, Grof P The developmental trajectory of bipolar disorder. Br J Psychiatry 2014; 204 122-8.

14 Machado-Vieira R, Luckenbaugh DA, Soeiro-de-Souza MG, Marca G, Henter ID, Busnello JV, et al. Early improvement with lithium in classic mania and its association with later response. J Affect Disord 2013; 144: 160-4.

15 McMahon FJ, Insel TR. Pharmacogenomics and personalized medicine in neuropsychiatry. Neuron 2012; 74: 773-6.

16 Chen $\mathrm{C}-\mathrm{H}$, Lee $\mathrm{C}-\mathrm{S}$, Lee M-TM, Ouyang W-C, Chen $\mathrm{C}-\mathrm{C}$, Chong $\mathrm{M}-\mathrm{Y}$, et al. Variant GADL1 and response to lithium therapy in bipolar I disorder. N Engl J Med 2014; 370: 119-28.

17 Hou L, Heilbronner U, Rjetschel M, Kato T, Kuo PH, McMahon FJ, et al. Variant GADL1 and response to lithium therapy in bipolar I disorder. N Engl J Med 2014; 370: 1857-9.

18 Ikeda M, Kondo K, Iwata N. Variant GADL1 and response to lithium therapy in bipolar I disorder. N Engl J Med 2014; 370: 1856-7.

19 Cipriani A, Hawton K, Stockton S, Geddes JR. Lithium in the prevention of suicide in mood disorders: updated systematic review and meta-analysis. BMJ 2013; 346: f3646.

20 Martinsson L, Wei Y, Xu D, Melas PA, Mathé AA, Schalling M, et al. Long-term lithium treatment in bipolar disorder is associated with longer leukocyte telomeres. Transl Psychiatry 2013; 3: e261.

21 McKnight RF, Adida M, Budge K, Stockton S, Goodwin GM, Geddes JR. Lithium toxicity profile: a systematic review and meta-analysis. Lancet 2012; 379: 721-8.

22 Malhi GS, Tanious M, Das P, Berk M. The science and practice of lithium therapy. Aus NZ J Psychiatry 2012; 46: 192-211.

23 Singh N, Halliday AC, Thomas JM, Kuznetsova OV, Baldwin R, Woon ECY, et al. A safe lithium mimetic for bipolar disorder. Nat Commun 2013; 4: 1332.

\section{Out-patient institutionalisation}

Nicholas Kontos, Oliver Freudenreich and John Querques

The term institutionalisation describes dehumanising processes by which people with mental illness were assimilated into the workings of large, long-term psychiatric hospitals. Patients were pushed further into deviance by role expectations imposed on them by the asylums' operational needs, intellectual assumptions and power dynamics. Contemporary clinic structures, 'checklist' driven interviews and psychiatric interventionism can induce behaviours in the community analogous to those observed in 'total institutions'. Many patients now relay and understand their psychological experiences solely through pathologising jargon (e.g. 'my depression,' 'my bipolar'). As soon as a patient is asked, 'Are you depressed?' role assumptions and expectations start to concretise. 